\title{
Reviewers in 2020
}

(c) The Japan Society of Brain Tumor Pathology 2020

Brain Tumor Pathology Editorial Office wishes to thank the following reviewers (non-editorial board members) for their contributions in 2020 .

Yonehiro Kanemura

Yukiko Shishido-Hara

Publisher's Note Springer Nature remains neutral with regard to jurisdictional claims in published maps and institutional affiliations. 